\section{Centrum für Integrative Psychotherapie (CIP)}

Supervisorenausbildung und CO-Supervision

Verhaltenstherapie/kognitiv-behaviorale Psychotherapie

Organisationsleitung: Dr. Dr. S. Sulz

Kursleitung: Prof. F. Kanfer, Dr. D. Schmelzer, Dr. Sulz unter anderem

2-jährige Ausbildung zum/zur Supervisor/in für Ärzte/innen und Diplom-Psychologen/innen mit abgeschlossener Ausbildung (Facharzt/ärztin für Psychiatrie und Psychotherapie bzw. für Psychotherapeutische Medizin bzw. psychologische/r Psychotherapeut/in - verhaltenstherapeutisch orientiert)

Beginn: Sommer 2001

Umfang:

ein viertägiges Start-Seminar (Donnerstag bis Sonntag)

sechs dreitägige Seminare (Freitag bis Sonntag) und zuletzt ein zweitägiges Abschlußseminar

(insgesamt 192 Stunden)

\section{Kosten:}

DM 34,- je Unterrichtseinheit (Gesamtkosten 6528,- DM)

Ziel: Qualifizierung in Einzel- und Gruppensupervision, unter anderem eigene Schwächen und Stärken im therapeutischen Bereich erkennen und sich weiterentwickeln, um Supervisanden in möglichst vielen therapeutischen Bereichen helfen zu können, Team-Supervision, Institution in/von Institutionen.

Inhalt: Therapie und Supervision im Live-Prozeß mit TriadenArbeit (Live-Selbstmodifikation, Live-Therapie, Live-Supervision), Video-Supervision, Co-Supervision, Supervision der Supervision. Eigene Dozententätigkeit, deren Supervision. Zwischen den Kursblöcken werden eigene Therapien, je einer der obigen Grundkonzeptionen folgend, mit Video-Dokumentation durchgeführt. Bei einem erfahrenen Supervisor erfolgt eine Co-Supervision. Außerdem werden zwischen den Blöcken Einzel- und Gruppensupervisionen selbst gehalten. Eigene Dozententätigkeit als Verhaltenstherapie-Kursleiter während des Kurses und zwischen den Kursblöcken mit didaktischem Feedback und Optimierung des Dozentenverhaltens.

$\mathrm{Zu}$ den Workshops werden wechselnde Spezialisten als externe Dozenten/Supervisoren eingeladen.

Zertifikat: Durch ein Abschlußkolloquium kann ein qualifizierendes Zertifikat erworben werden, das nach Ablauf der Mindestzeit bei einem öffentlich-rechtlichen Gremium eingereicht werden kann.

Information und Beratung bzw. noch mehr Information: CIP, Nymphenburgerstr. 185, 80634 München
Tel.: 089/13079315 (Montags 11.00 - 12.00 Uhr)

Bitte senden Sie Ihr Bewerbungsschreiben an Dr. Sulz, CIP, Nymphenburger Str. 185, 80634 München

Legen Sie dazu:

Psychologie-Diplom und psychotherapeutische Approbation oder ärztliche Approbation

Zeugnis Ihres Verhaltenstherapie-Ausbildungsabschlusses

- Curriculum vitae eventuell mit Publikationen

- kurze Beschreibung Ihres gegenwärtigen Tätigkeitsfeldes

- Lichtbild

Achtung: Sie brauchen 1 Videokamera, um Ihre Therapien und Supervisionen aufnehmen zu können (am besten S-VHSC mit 45-Minutenbändern). Wenn Sie eine Video 8-Kamera haben, müssen Sie zuhause Ihr Video 8-Band zuerst auf eine normal große VHS-Cassette überspielen, damit wir sie im Kurs abspielen können. Dagegen kann das Band von Ihrer VHS-C-Kamera direkt bei uns abgespielt werden (Adapter vorhanden). Auf Ihr Video als Kursmedium können wir auf keinen Fall verzichten.

\section{Institut für Verhaltenstherapie und Verhaltensmedizin (IVV) e.V.}

\section{Ziele}

Ausbildung in Verhaltenstherapie und verhaltenstherapeutische Öffentlichkeitsarbeit

Ausbildung in Verhaltenstherapie

Das IVV ist ein anerkanntes psychotherapeutisches Ausbildungsinstitut am Klinikum der Philipps-Universität Marburg, das von Psychologen und Medizinern in enger Kooperation mit den Universitätskliniken für Psychiatrie und Psychotherapie sowie für Psychiatrie und Psychotherapie des Kindes- und Jugendalters gegründet wurde und geleitet wird. Das IVV bietet eine umfassende Vollzeitausbildung (3-jährig) in Verhaltenstherapie und Verhaltensmedizin an:

- Für Psychologen zur Approbation als Psychologischer Psychotherapeut

- Für Psychologen und Pädagogen zur Approbation als Kinder- und Jugendlichen-Psychotherapeut

- Für Ärzte zum Erwerb des Zusatztitels Psychotherapie (Verhaltenstherapie)

- Für Ärzte die zum Erwerb des Facharztes für Psychiatrie und Psychotherapie oder des Facharztes für Kinder- und Jugendpsychiatrie und -psychotherapie notwendigen Qualifikationsbestandteile auf den Gebieten der Verhaltenstherapie und Verhaltensmedizin 
Der Status als Vollzeitausbildung ermöglicht die Beantragung von BAföG-Förderung.

Die Ausbildung am IVV läuft seit Frühjahr 2001. Dozenten des IVV in den Ausbildungsjahren 2000 und 2001 waren und werden unter anderem Prof. H. Reinecker, Prof. S. Gauggel, Prof. W. Hiller, PD Dr. R. Lässle, Prof. Dr. Dr. H.D. Basler, Prof. Dr. G. Esser, PD Dr. M. von Aster, Prof. Dr. J. Margraf und Dr. S. Schneider.

Der nächste Kurs am IVV beginnt im Herbst 2001.

Verhaltenstherapeutische Öffentlichkeitsarbeit

Das IVV veranstaltete bisher

- Marburger Verhaltenstherapietag (1999)

- Marburger Verhaltenstherapietag (2000)

- Schmerztherapie-Workshop von Prof. F. J Keefe (Durham, USA; 2000)

- Depressions-Telefonaktion und -Sprechstunde (2000)

Das IVV plant in Zusammenarbeit mit der Philipps-Universität Marburg und dem Universitätsklinikum, dem Marburger Universitätsbund, dem Kompetenznetzwerk «Depression» und der Stadt Marburg weitere Aufklärungsaktionen und Präventionsmaßnahmen zur Bekämpfung depressiver Erkrankungen.

\section{Fortbildungsinstitut für Klinische Verhaltenstherapie}

\section{Weiterbildungsveranstaltungen 2001}

Das Fortbildungsinstitut für Klinische Verhaltenstherapie veranstaltet regelmäßige Workshopangebote in der Regel über 16 Unterrichtseinheiten. Ziel dieser Workshops ist es, den Teilnehmern die Möglichkeit zu geben, gemeinsam mit namhaften Referenten aktuelle Weiterentwicklungen der Verhaltenstherapie kennenzulernen oder andererseits auch Kontakt mit Vertretern anderer Therapieschulen zu erhalten.

Die Workshops des Fortbildungsinstitutes für klinische Verhaltenstherapie werden im Rahmen des ärztlichen Weiterbildungsrechts mit Akkreditierungspunkten versehen. Bitte erkundigen Sie sich im Vorfeld, ob die von Ihnen gewünschte Veranstaltung die entsprechenden Bedingungen erfüllt.

\begin{tabular}{ll} 
Datum Thema $\quad$ Dozent \\
\hline
\end{tabular}

11./12.05.2001 Verhaltenstherapie bei Johann Vanderlinden, Beginn Freitag! Eßstörungen Dipl.-Psych. Universitäts-Centrum St. Jozef, Kortenberg, Belgien

07./08.09.2001 Verhaltenstherapie bei Christina Wiesemann Beginn Freitag! Persönlichkeitsstörungen Dipl.-Psych. München 27./28.10.2001 Kognitive Verhaltens- $\quad$ PD Dr. med. Ulrich therapie bei depressiven Schweiger Störungen

\begin{tabular}{lll} 
Datum & Thema & Dozent \\
\hline $\begin{array}{ll}\text { 30.11./01.12.2001 } \\
\text { Beginn Freitag! }\end{array}$ & Kopfschmertenstherapie bei & $\begin{array}{l}\text { Professor Dr. rer. soc., } \\
\text { med. habil. Wolfgang }\end{array}$ \\
& H. R. Miltner \\
& Lehrstuhl f. Biologische u. \\
& Klinische Psychologie, \\
& Institut für Psychologie \\
& Friedrich-Schiller- \\
& Universität Jena
\end{tabular}

Ort: Psychosomatische Fachklinik Bad Pyrmont, Bombergallee 10, 31812 Bad Pyrmont, Tel. 05281/6190

Beginn der Veranstaltungen jeweils Samstag 10.00 Uhr (Ausnahme: der Workshop am 11./12.05.2001, 07.08.09.2001 und 30.11./01.12.2001 beginnt Freitag um 16.00 Uhr)

Anmeldungen bitte schriftlich oder telefonisch unter:

FIKV, Bombergallee 11, 31812 Bad Pyrmont, Tel. 05281/ 606763 oder 619636 per E-Mail: sekretariat@fikv.de

Sie finden das Programm auch im Internet unter: www.fikv.de oder auf der Homepage der Psychosomatischen Fachklinik unter: www.fkbp.de

Die Teilnahmegebühr beträgt DM 320,00.

Die Gebühren werden mit der Anmeldung fällig. Bei Platzrückgabe bis drei Wochen vor der Veranstaltung wird eine Stornogebühr von DM 32,00 fällig. Bei späterer Stornierung wird die Kursgebühr in voller Höhe fällig. Eine Gebührenermäßigung kann in Einzelfällen gewährt werden.

\section{Deutsche Gesellschaft für Verhaltenstherapie e.V. (DGVT)}

Bundesgeschäftsstelle:

Neckarhalde 55, 72072 Tübingen, Tel. 07071/9434 -0

E-mail-Adresse: dgvt@dgvt.de, Internet: www.dgvt.de

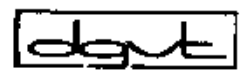

Deutsche Gesellschaft für Verhaltenstherapie e.V. (DGVT) ruft in Zusammenarbeit mit der

Gesellschaft für Gemeindepsychologische Forschung und Praxis (GGFP) auf zur Mitarbeit am

14. Kongress für Klinische Psychologie, Psychotherapie und Beratung

vom 17. - 22. Februar 2002 in Berlin

Die Deutsche Gesellschaft für Verhaltenstherapie und die Gesellschaft für Gemeindepsychologische Forschung und Praxis laden ein, den 14. Kongress für Klinische Psychologie, Psychotherapie und Beratung mitzugestalten. Die Gesellschaft richtet in den letzten Jahren immer größere (Heils-) Erwartungen an die Genforschung und die Biotechnologie mit der Konsequenz, dass die psychosoziale Ebene von Gesundheit und Krankheit vernachlässigt wird. Der 14. Kongress für Klinische 
Psychologie, Psychotherapie und Beratung soll daher unter dem Schwerpunktthema

\section{Gentherapie statt Psychotherapie? (K)Ein Abschied vom Sozialen?}

stehen. Mit dieser - sicherlich pointierten - Fragestellung sollen die Sozialwissenschaften, zu denen auch die Klinische Psychologie, die Psychotherapie und die Gemeindepsychologie zählen, ihr Verhältnis zur Genforschung, Biotechnologie und somatischen Medizin bestimmen. Für den Kongress werden Veranstaltungen geplant, die die Möglichkeiten und Grenzen der Gendiagnostik und -therapie sowie der Biotechnologie vorstellen sollen. Das Schwerpunktthema soll anregen, das Verhältnis von Sozialwissenschaften, klinischer Psychologie, Psychotherapie, Beratung, Gemeindepsychologie und deren Praxis zur Genforschung und Biotechnologie zu bestimmen. Beispielhaft seien hier Themen für mögliche Symposien benannt:

- Genforschung und Bioethik: sozialwissenschaftlich begründete Perspektiven

- Genforschung und Biotechnologie - die Machbarkeit eines «neuen» Menschen?

- Die genetischen Grundlagen psychischer Empfindungen Forschungsergebnisse der Verhaltensgenetik

- Konsequenzen der Genforschung: Die psychische Bewältigung des neuen Wissens - oder: neue Anforderungen an die Beratungsarbeit

- Pränatale Genanalyse - oder (k)ein Recht auf ein behindertes Leben?

- Selektionsvorteile genetischer Defekte - oder: Was kann die Gendiagnostik leisten?

- Der Mensch als biotisches Wesen in seiner sozialen Verfasstheit oder: gegen einen Determinismus der Gene

- Die psychische Bewältigung von gendiagnostischen und gentherapeutischen Behandlungen - Aufgaben der Beratung und der Psychotherapie

Dies sind nur einige der möglichen, denkbaren Themen, die aus dem Spannungsbogen Genforschung/Biotechnologie und sozialwissenschaftlich-orientierter Psychologie entstehen.

Selbstverständlich bietet auch der 14. Kongress für Klinische Psychologie, Psychotherapie und Beratung Raum für die «traditionellen» Kongressthemen der DGVT. Auch hier seien beispielhaft einige Themenfelder benannt:

- Klinisch-psychologische Forschungsthemen wie

- Psychotherapieprozessforschung

- Evaluationsmethoden in der Klinischen Psychologie

- Evaluationsmethoden in der Psychotherapeutischen Praxis

- Indikationsmodelle in der Psychotherapie

- Methodenforschung

- Integrationsmodelle

- Entwicklungen der Verhaltensmedizin

- Entwicklung der Allgemeinen Psychologie
- Störungsspezifische Symposien zu verschiedenen Störungs- und Krankheitsbildern (wie zum Beispiel: Panikattacken, Schizophrenieforschung, Depressionen, psychosomatische Störungen) sowie

- die Weiterführung von Themen, die auf den letzten Kongressen Schwerpunktthema waren.

- Gemeindepsychologie und Beratung

- Neue Beratungsstrategien

- Beratung in verschiedenen Tätigkeitsfeldern wie Schule, Erziehung, Organisation und Institutionen

- Gesundheitsförderung und

- Möglichkeiten der primären Prävention

- Rehabilitationsforschung

- vernetzte Strukturen in der psychotherapeutischen Arbeit

- Erste Erfahrungen mit der Integrierten Versorgung nach $\S 140$ SGB V

- Entwicklung in der Psychiatriereform

- Partizipationsprozesse in der Psychotherapie und der psychosozialen Praxis

- Gesundheitspolitische Themen

Bei der Organisation der Symposien ist darauf zu achten, dass das jeweilige Thema möglichst aus unterschiedlichen Perspektiven bearbeitet und dargestellt wird. Dazu gehört insbesondere

- die geschlechtsspezifische Perspektive

- die Perspektive von Migrantinnen und Migranten und

- der Blick der Nutzerinnen und Nutzer.

Der 14. Kongress für Klinische Psychologie, Psychotherapie und Beratung wird die bewährten Methoden der Wissensvermittlung - wie Referat, Workshop und Poster - nutzen und fortentwickeln. Alle, die sich an dem Kongress beteiligen, sind aufgerufen, mit neueren Methoden den Kongress mitzugestalten und somit die bewährte DGVT - Kongressatmosphäre zu schaffen.

Sie sind herzlich eingeladen, sich an der inhaltlichen Ausgestaltung des Kongresses zu beteiligen. Sie können Ihr Interesse an der Organisation und Durchführung eines Beitrages dazu gehört die Organisation eines Symposiums, eines Referates, eines Workshops, einer Arbeitsgruppe oder eines Posters - bei der

DGVT Bundesgeschäftsstelle, Postfach 1343, 72003 Tübingen unter dem Stichwort: Kongress 2002 anmelden.

Falls Sie ein Symposium organisieren möchten, schicken Sie uns Ihre Vorstellungen bis Ende März 2001, für die anderen Angebote (siehe unten) ist der Einsendeschluss Ende Juni. Bitte skizzieren Sie den Inhalt Ihres Beitrages in einigen Sätzen (max. auf einer Schreibmaschinenseite). Stellen Sie insbesondere dar, ob Sie beabsichtigen, ein Referat, ein Poster, einen Workshop zu halten oder ob Sie bereit sind, ein Symposium zu einer spezifischen Fragestellung zu organisieren. Bei der Planung eines Symposiums achten Sie bitte darauf, dass dieses möglichst nicht länger als 4 Stunden dauern sollte. 
Sie können Ihren Beitrag auch per Internet an die DGVTBundesgeschäftsstelle weiterleiten. Ein entsprechendes Formblatt finden Sie auf der Homepage der DGVT unter www.dgvt.de

\section{SYMPOSIUM \\ Die Präsentation eines Themas mit bis zu 4 Referaten Dauer: In der Regel 4 Stunden \\ 2. POSTER \\ 3. REFERAT \\ max. 20 minütiger Vortrag (möglichst mit Folien) \\ 4. WORKSHOP \\ Vermittlung von praktischen Fertigkeiten in vier Stunden \\ 5. ARBEITSGRUPPE \\ Gruppenarbeit zu einem Thema mit einem Impulsreferat}

Weitere Informationen erhalten Sie im

Kongressreferat, Neckarhalde 55, 72070 Tübingen

Tel.: 07071/94 34 94, Fax: 07071/94 34 35, www.dgvt.de, dgvt@dgvt.de

\section{Fortbildungen der GAP}

01.06.-02.06.01 Noncompliance und Widerstand in der

Frankfurt Verhaltenstherapie

Deutschland

Dr. med. Dipl.-Psych. Raimund

Dankesreiter

12.10.-13.10.01

Frankfurt

Prävention und Bewältigung von

Deutschland Problemen in Expositionsbehandlungen

Auskunft: $\quad$ GAP - Gesellschaft für Ausbildung in Psychotherapie

Dipl.-Psych. Christian Alte

Beethovenstr. 18, 60325 Frankfurt

Tel.: 069 / 7411 888, Fax 069 / 7403 62,

E-mail: info@gap-ffm.de

\section{Aus- und Weiterbildungsangebote des IFKV Bad Dürkheim}

Staatlich anerkanntes Ausbildungsinstitut für die Ausbildungsgänge:

Psychologische/r Psychotherapeut/in

Kinder-/ und Jugendlichenpsychotherapeut/in

Das IFKV bietet folgende Ausbildungsgänge (Verhaltenstherapie) nach dem Psychotherapeutengesetz an:

Psychologische/r Psychotherapeut/in

Kursbeginn: Frühjahr und Herbst eines Jahres

Kinder- und Jugendlichenpsychotherapeut/in

Kursbeginn: Mai 2001

Darüber hinaus finden Weiterbildungskurse in folgenden

Bereichen statt:

Verhaltenstherapeutische Supervision:

Kursbeginn: September 2001

Verhaltenstherapeutische Cotherapie:

Kursbeginn: November 2001

Kinder- und Jugendlichen-Zusatz-Weiterbildung:

Kursbeginn: Herbst 2001

Ärztliche Weiterbildungsangebote:

auf Anfrage

Fortbildungswoche vom 07.05. bis 13.05.2001

Im Rahmen der Nachqualifikation findet am IFKV vom 07.05. bis 13.05.2001 eine Fortbildungswoche im Umfang von 56 Unterrichtseinheiten zu folgenden Themen statt: Gruppentherapie, ADD bei Erwachsenen, Fallarbeit, Erstellung von Kurz-/Langzeitanträgen, Somatische Differentialdiagnose und Somatisierungsstörungen.

Fortbildungstage 2001:

23.06.2001 EMDR mit Christof T. Eschenröder

01.12.2001 FOCUSING mit Johannes Wiltschko

Fachtagung

Die Verhaltenstherapie und der Körper - eine Beziehung mit Zukunft?

am 27. Oktober 2001 in Bad Dürkheim

Anlässlich des 15-jährigen Bestehens des IFKV im Jahr 2001 plant das Institut in Kooperation mit der ParkKlinik Bad Bergzabern, Rehabilitationszentrum für Psychosomatik, eine eintägige Fortbildungsveranstaltung zum Thema «Verhaltenstherapie und Körpertherapie». Vorträge und Workshops werden einen Überblick über gängige Verfahren geben. Verschiedene Ansätze sollen im Hinblick auf ihre Anwendbarkeit bzw. Adaption für die VT-Praxis diskutiert und ausprobiert werden. Am Abend gibt es dann beim anschließenden Tagungsfest Gelegenheit, die neuen Erfahrungen umzusetzen.

Informationen:

Institut für Fort- und Weiterbildung in klinischer

Verhaltenstherapie e. V.

Kurbrunnenstr. 21 a, 67098 Bad Dürkheim

Tel. (0 63 22) 680 19, Fax: (0 63 22) 68010

E-mail: info@ifkv.de

Internet: http://www.ifkv.de 